\title{
Unilateral Pleural Effusion in Grave's Thyrotoxicosis; A Case Report
}

\author{
Amr W Aljareh, MD, MRCP, Mohammed H Lhmdi, MD, Safia Inam, MD, \\ Zainab Alsharef, MD, Walid Hassan, MD, FAHA, FACC, FCCP, FACP*
}

Department of Cardiovascular Disease, International Medical center, Jeddah, Saudi Arabia *Professor of Medicine and Senior Consultant, Director of the Cardiac Center of Excellence International Medical center, Sa udi Arabia whassan@imc.med.sa

\begin{abstract}
In this report, we introduce a case of a middle aged patient who was admitted and diagnosed as Graves' disease; the case was associated with unilateral (right) pleural effusion which could be a rare complication of Graves' disease. As it is rarely reported and not widely known, this association may be under diagnosed and we suggest that Grave's hyperthyroidism should be considered in the differential diagnosis of pleural effusion in patients with thyrotoxicosis.
\end{abstract}

\section{INTRODUCTION}

Pleural effusion is a common problem dealt by most of the practicing clinicians. Some causes for pleural effusion are less often considered as a differential diagnosis owing to its rarity (1). Effusions (pleural and pericardial) are rare in hyperthyroidism as compared to hypothyroidism and predominantly reported in patients with Grave's disease (2).

In hyperthyroidism, accelerated thyroid function results in secretion of excessive amount of thyroid hormones. The most common symptoms are weight loss, irritability, heat intolerance, sweating, fatigue, weakness, gastrointestinal hypermobility, and diarrhea. Common signs are tachycardia, atrial fibrillation, congestive heart failure, tremor, goiter, warm moist skin, and muscle weakness (3).

Herein, we present the case of a 43 year old man who had unilateral pleural effusion associated with thyrotoxicosis.

\section{CASE PRESENTATION}

This is a 43 years old Filipino male came with 3 months history of unintentional weight loss $>15 \mathrm{~kg}$, and watery diarrhea for 3 months, 4 motions a day with no blood and no mucus. The patient also had history of dyspnea and Palpitation for 3 months.

Review of systems was unremarkable with no history of fever, chest pain, heat intolerance, sweating or restlessness.

There was no relevant past medical, family, social or allergic history and had not been taking any medication before hospital admission.

On admission, his physical examination was normal, with a blood pressure of $119 / 79 \mathrm{mmHg}$ and a pulse rate 113 beats/ min with irregular rhythm. respiratory rate 20 cycle/min and Temperature 36.5 C. saturation $94 \%$ on air room.

There is increase in the eyeball size bilaterally (exophthalmoses). His neck examination showed no nodular thyroid or thyroid masses. There was no lymphadenopathy. Dullness to percussion was found over the lower portion of her right hemithorax, accompanied by decreased vocal fremitus, as well as decreased breath sounds on auscultation. The chest examination was normal over his left hemithorax. Cardiac examination revealed no murmurs, rubs, or gallops, but heart sounds were irregular and distant. Abdominal examination was unremarkable, with no signs of ascites or masses. Lower extremities revealed intact pulses with no edema. 
Electrocardiography shows atrial fibrillation with no other significant findings. Chest $\mathrm{x}$ ray showed moderate right sided pleural effusion with cardiomegaly (Figure 1).

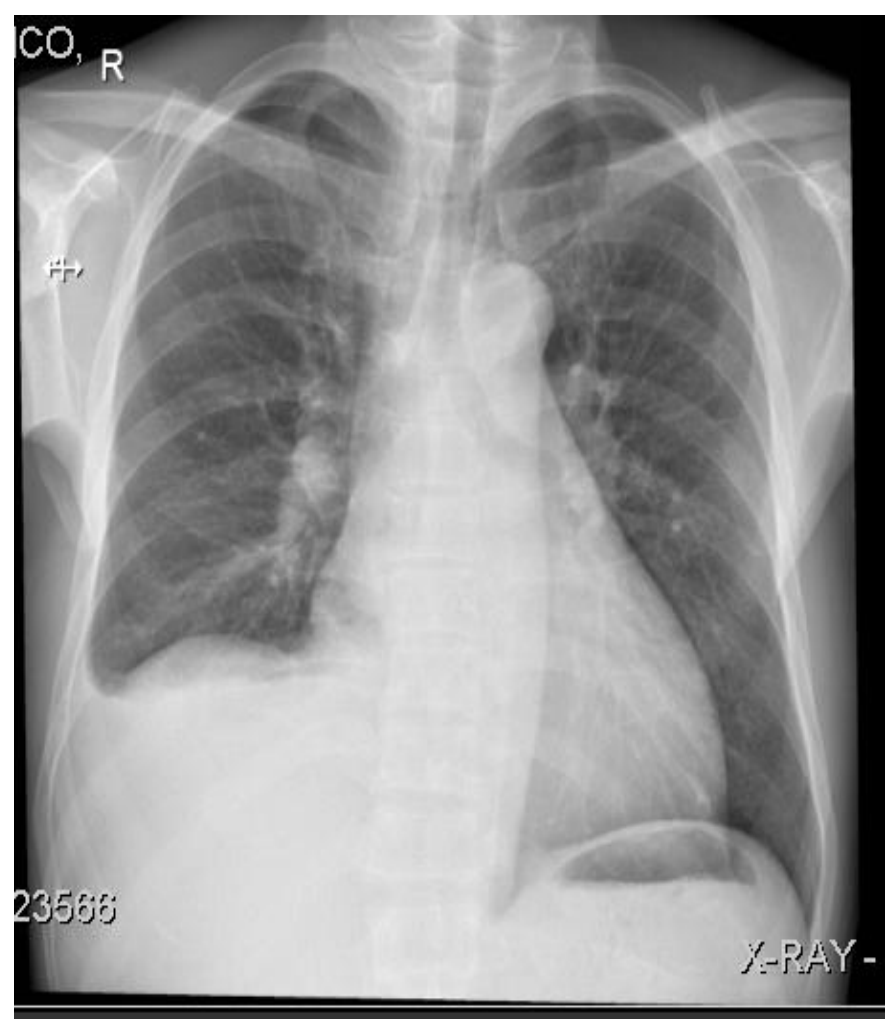

Figure1. Chest $x$ ray

Results of blood analysis were normal except for thyroid function: the thyroid-stimulating hormone (TSH) level was 0.01 $\mu \mathrm{IU}$ (Reference range: $0.27-4.20 \mu \mathrm{IU}), \quad \mathrm{T} 4: 78 \mathrm{pmol} / \mathrm{L}$ (reference range (RR): 12.0-22.0 pmol/L) and TSIG: 28.2

Technetium 99-m thyroid gland scintigarphy showed diffuse goiter with high uptake finding ongoing with the diagnosis of hyperthyroidism due to Graves' disease (Figure 2).

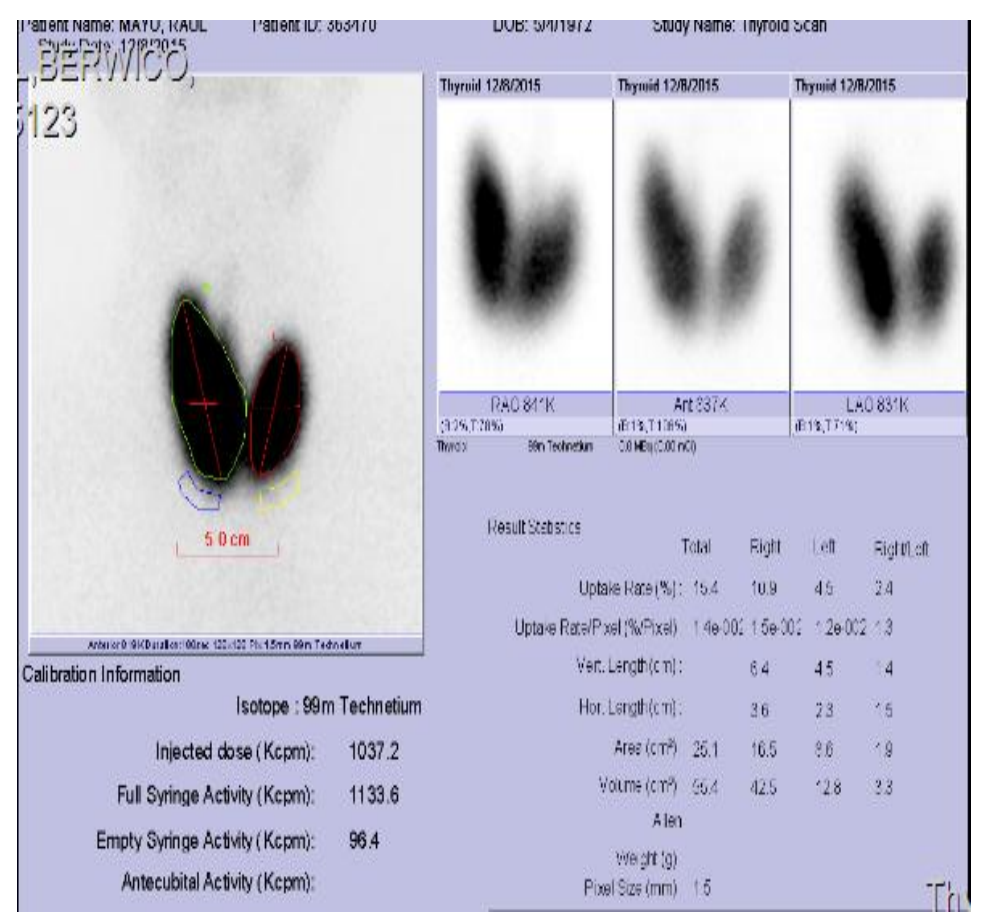

Figure2. Technetium 99-m thyroid gland scintigarphy

Chest and abdomen CT scan showed the right pleural effusion but no evidence of intrathoracic or abdominal malignancy (Figure 3). 


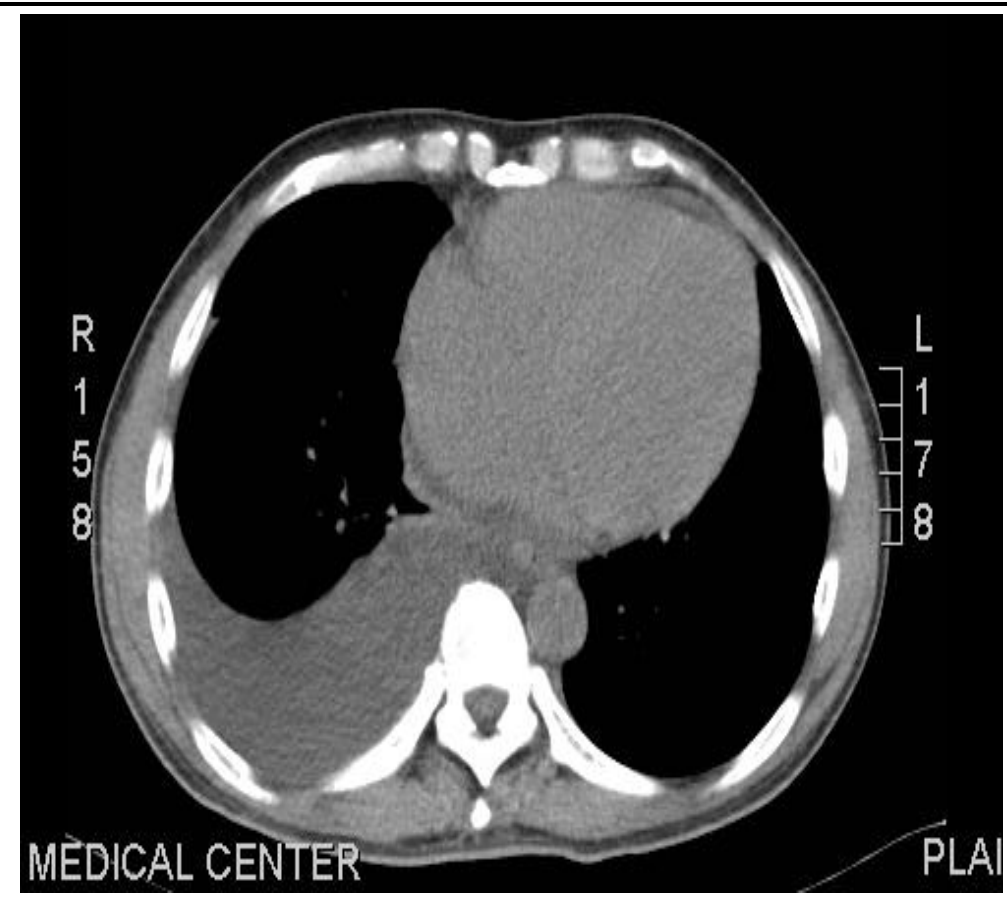

Figure3. Chest CT scan

Echocardiogram was done and revealed good left ventricle systolic function, normal ejection fraction, mild left atrium dilation, and small pericardial effusion (Figure 4).

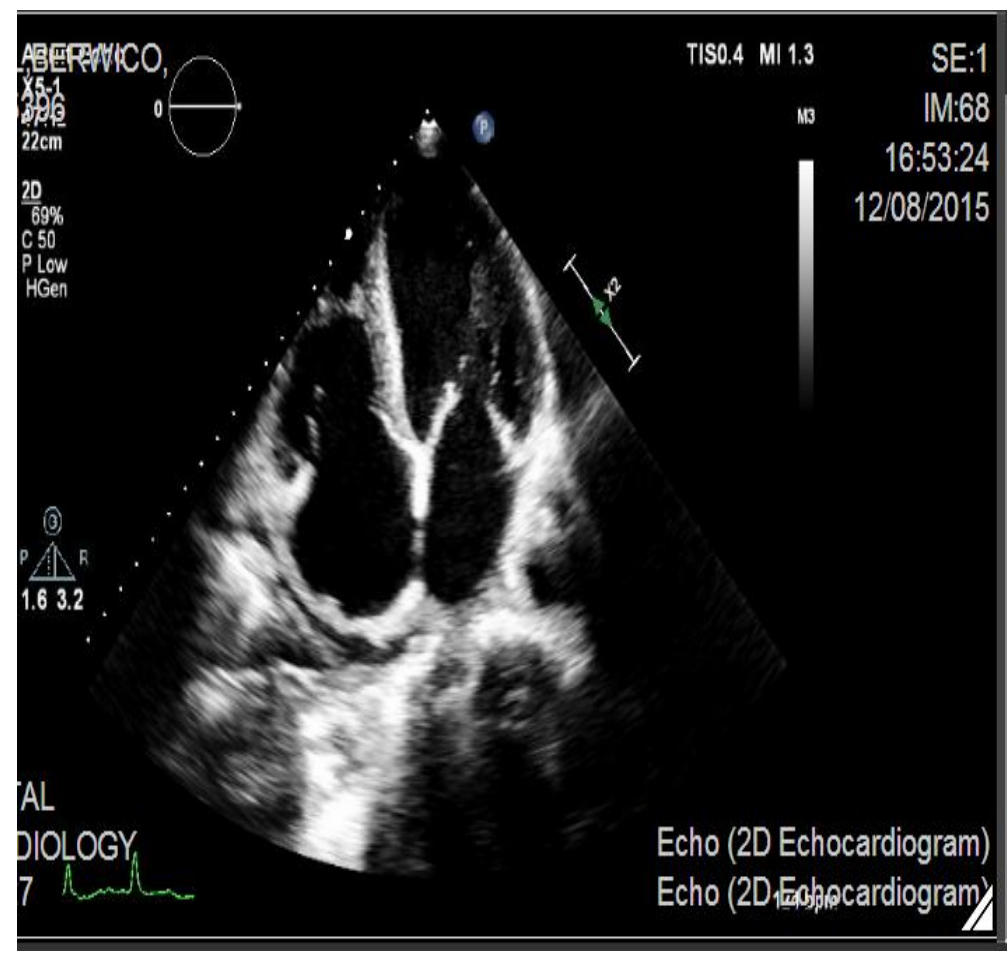

Figure4. Echocardiogram

The patient was admitted under cardiology team care as a case of hyperthyroidism complicated with atrial fibrillation and pleuropericardial effusion.

The patient was started on Propranolol $40 \mathrm{mg}$ three times daily, ASPIRIN $81 \mathrm{mg}$ daily and low molecular weight heparin (clexan) 60 subcutaneous twice daily.

Endocrinology team started radioactive iodine with much improvement of his symptoms and follow up chest $\mathrm{x}$ ray and echocardiogram after 4 weeks showed complete resolution of his pleural and pericardial effusion (Figure 5). He remains euthyroid and asymptomatic for one year. 


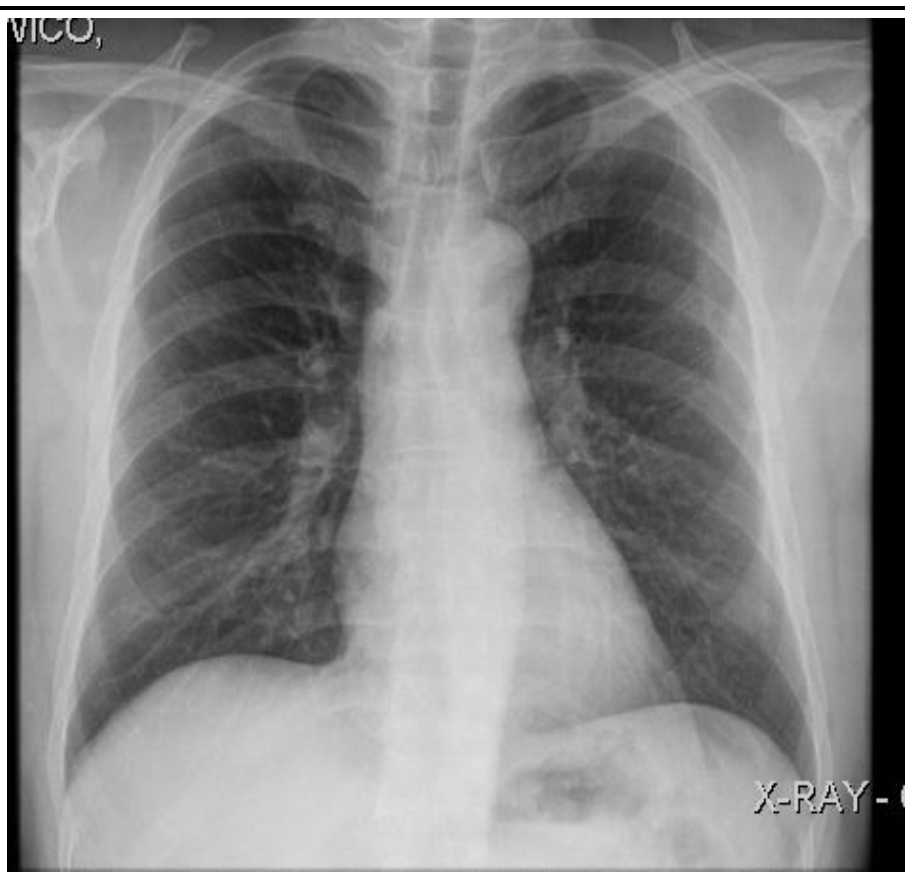

Figure5. Chest $x$ ray- Follow up

\section{DISCUSSION}

Pleural effusions can develop as a result of over 50 different pleuropulmonary or systemic disorders. Following diagnostic thoracentesis, the cause of a pleural effusion is not evident in up to 25 percent of patients $(4,5)$. However, no universally accepted definition exists for an "undiagnosed effusion."

In our patient there were several possible explanations for pericardial and pleural effusions. Heart failure in patients with hyperthyroidism and atrial fibrillation can cause effusions, although normal left ventricular function on echocardiogram and normal cardiac biomarkers did not support this diagnosis. Immune-mediated conditions like systemic lupus erythematous are another potential explanation in a patient with autoimmune thyroid disease but this was excluded by the absence of suggestive clinical features and laboratory analysis. Primary or secondary malignancy and infection also could be a potential cause but, were excluded by absence of suggestive clinical picture, intrathoracic and intrabdomenal CT scan and her subsequent clinical course. We therefore believe that the effusion was caused by Graves' disease, and the underlying pathophysiology could be secondary to immunological mechanism similar to that of pretibial, preorbital myxedema and ophthalmic myopathy.

There are many cases of pericardial and pleural effusion in the setting of hypothyroidism but only very few case reports in the literature which have described association of pleural and pericardial effusions with Graves' hyperthyroidism $(6,7)$.

In the hyperthyroidism case reports, none of the patients required any treatment other than antithyroid drugs with rapid improvement in the patients' general condition (6).

Our patient was treated with B-blocker for the atrial fibrillation and radioactive iodine only with complete resolution of his effusion without the need for therapeutic taping.

So we suggest that Grave's hyperthyroidism should be considered in the differential diagnosis of pleural effusion in patients with thyrotoxicosis and analysis of more case is needed to better understand the underlying pathophysiology of this association.

\section{Conclusion}

Pleural and pericardial effusions are rare but recognized complications of thyrotoxicosis and

Graves' disease and should be considered in patients who present with dyspnea, atrial fibrillation.

So we suggest that Grave's hyperthyroidism should be considered in the differential diagnosis of pleural effusion in patients with thyrotoxicosis and analysis of more case is needed to better understand the underlying pathophysiology of this association. 


\section{REFERENCES}

[1] George S, Ravindran M, Anandan P T, Kiran V N. Primary systemic amyloidosis: A rare cause for pleural effusion. Respir Med Case Rep 2014; 13: 39-42.

[2] Khalid Y, Sulaiman R, Zahir R, Baskar V, Buch H N. An unusual complication in a patient with Graves' disease. New Zealand med J. 2011; 124: 1340.

[3] Papadakis M A, McPhee S J, Rabow M W. Hyperthyrodism in: Current medical diagnosis and treatment. Fifty fourth edition. USA: McGraw Hill; 2015. p. 1099- 1108.

[4] Collins TR, Sahn SA. Thoracentesis. Clinical value, complications, technical problems, and patient experience. Chest 1987; 91 (6): 817-22.

[5] Venekamp LN, Velkeniers B, Noppen M. Does 'idiopathic pleuritis' exist? Natural history of non-specific pleuritis diagnosed after thoracoscopy. Respiration 2005; 72:74.

[6] Clarke NR, Banning AP, Gwilt DJ, Scott AR. Pericardial disease associated with Grave's thyrotoxicosis. QJM 2002; 95(3):188-189.

[7] Nakata A, Komiya R, Ieki Y, Yoshizawa H, Hirota S, Takazakura E. A patient with Graves' disease accompanied by bloody pericardial effusion. Internal Medicine. 2005; 44(10):10641068 . 